\title{
A Cyber-Enabled Stabilizing Controller for Resilient Smart Grid Systems
}

\author{
Abdallah K. Farraj, Eman M. Hammad, and Deepa Kundur \\ Department of Electrical and Computer Engineering, University of Toronto, Ontario, Canada \\ Email: \{abdallah, ehammad, dkundur\}@ece.utoronto.ca
}

\begin{abstract}
A parametric controller is proposed for the frequency and phase stabilization after the occurrence of a disturbance in the power grid. The proposed controller is based on the feedback linearization control theory. To drive the frequency of the system generators to stability, the controller relies on receiving timely phasor measurement unit (PMU) readings about the power grid to employ fast-acting flywheels that are situated near the synchronous generators in order to balance a swing equation model of the synchronous generators. The advantages of the proposed controller are that it is tunable and integrates well with existing governor controls in contrast to other forms of PMU-based control. Numerical results show the effectiveness of the proposed controller when applied to the New England power system. Further, a comparison is drawn between the controller and recently-proposed nonlinear controllers for transient stability.
\end{abstract}

\section{INTRODUCTION}

Smart grid systems employ control, communications, and sensor technologies to improve the reliability and efficiency of the power delivery system. Information about system operation is collected using sensors such as phasor measurement units (PMUs) placed in specific locations around the grid. The cyber data is then transmitted through a communication network to distributed controllers where it is analyzed to determine if any actions must be applied to enhance system operation and efficiency.

The introduction of advanced telemetry and communication technologies to the power systems opens the door to a variety of new nontraditional controller design opportunities. Moreover, controllers can be employed to improve resilience of the overall cyber-physical smart grid system. Recently, Wei et al. [1]-[3] employed the concept of flocking in multi-agent systems for the design of distributed controllers that aid in the transient stability of synchronous generators in the face of a severe disturbance. In the proposed framework, real-time PMU data is employed to compute control actions that are actuated via fast-acting energy storage systems.

Flocking-based control can be used in a power system to achieve transient stability through frequency synchronization and phase cohesiveness amongst synchronous generators. However, the controller can be costly computationally and demonstrates a graceful yet slow time scale for destabilization as communication latency grows. Consequently, questions arise as to whether more aggressive strategies exist that can drive the power system to stability in a shorter period of time.

Further, a recent work of Andreasson et al. [4]-[6] proposed using a consensus proportional integral (CPI) control scheme to affect the mechanical power of a generator in order to achieve an automatic frequency control strategy that is applied in two levels. The generator's frequency is controlled against a reference frequency in the first level, and the reference frequency is updated to eliminate errors in the second level. It is obvious that in order to find the value of the control output, the CPI controller needs to know the frequency of all system generators; in other words, it is a centralized controller.

This article proposes a low-complexity tunable distributed controller that easily integrates with generator governors. When the power system undergoes a transient instability, the proposed solution utilizes state information to execute a feedback linearization control scheme that leads the generators to stability more aggressively. Feedback linearization is a wellknown approach to convert a nonlinear system (plant) into an equivalent linear system through feedback control. One form of feedback linearization control involves canceling out the nonlinear terms in the plant such that the resultant dynamics of the closed-loop system is linear.

To achieve stability, sensor measurements are periodically communicated to the distributed controllers that then actuate change through fast-acting power injection and absorption entities such as flywheels. The actuation stabilizes the power grid by shaping the dynamics of the closed-loop system to resemble that of a series of stable decoupled linear systems with tunable eigenvalues. The short stability time makes the proposed controller a good candidate for distribution systems in which response time (say due to the presence of lower inertia renewable energy sources) must be short. Moreover, the controller integrates conveniently with other power system controls such as governors. The proposed solution does not need real-time information updates to practically stabilize the power system. Consequently, we assert that the introduction of such controller can enhance system resilience. Previously, feedback linearization was investigated in [7] to control the excitation system of the generators; however, our proposed solution utilizes external power sources to stabilize the frequency and achieve phase cohesiveness among the generators.

Contributions of this work include proposing a feedback linearization controller for smart grids that undergo a transient instability. Further, a simple, yet effective, formulation of phase cohesiveness is investigated. Moreover, the performance of the controller is compared to that of recent published work.

The rest of this article is organized as follows. The problem setting is presented in Section II and the proposed controller 


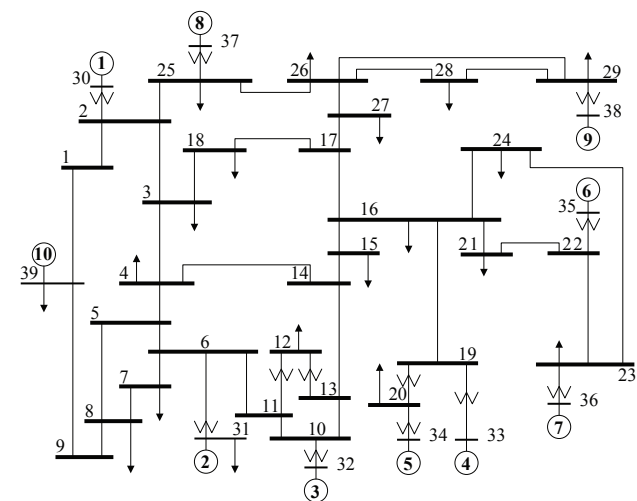

Fig. 1. New England power system

is detailed in Section III. Section IV investigates the controller performance. Conclusions are shown in Section V.

\section{Distributed Control Setting}

We assume that the smart grid is comprised of $N$ agents where each agent is comprised of a synchronous generator with an associated PMU that provides information on generator rotor angle and frequency, and a distributed controller that processes PMU data from the local and neighboring generators to actuate a local fast-acting power injection and absorption entity such as a flywheel. A communication network connects the PMUs and the distributed controllers.

The dynamics of each agent in the considered system depend on its own state and the states of other agents in the multi-agent system. In such setting, a centralized controller refers to a scheme where the states of all the agents in the system need to be collected; however, in a distributed scheme, the controller of a certain agent needs the state of that agent and its neighbors. Further, a decentralized controller requires only the state of its own agent.

The overall smart grid is a cyber-physical system in which the physical components correspond to the classical power delivery components as well as the fast-acting sources, and the cyber entities include the PMU sensors, distributed controllers and their associated communication network. The physicalto-cyber interface occurs at the sensors while the cyber-tophysical interface occurs at the controllers.

We consider the New England 10-generator 39-bus physical power system shown in Fig. 1. In this power system, Generator 10 at Bus 39 represents the aggregation of a large number of power generators. Let $N$ denote the number of generators in the power system (i.e., $N=10$ ). The parameters of the generators of the power system are defined in Table I and are expressed in per units, with the exception of $M_{i}$ and $D_{i}$ which are expressed in seconds, and $\delta_{i}$ which is expressed in radians.

We employ the swing equation model to describe physical synchronous generator dynamics. The rotor angle and frequency states of such a model ideally enable the study of transient stability or frequency stability. We assume that the swing equation parameters are constant even when the system undergoes instability. To address the physically networked nature of the power system, we make use of Kron-reduction to

\begin{tabular}{|c|l|}
\hline Parameter & Description \\
\hline$E_{i}$ & internal voltage of Generator $i, \forall i \in\{1, \ldots, N\}$ \\
$P_{e, i}$ & electrical power of Generator $i$ \\
$P_{m, i}$ & mechanical power of Generator $i$ \\
$\delta_{i}$ & rotor angle of Generator $i$ \\
$\omega_{i}$ & relative normalized rotor frequency of Generator $i$ \\
$X_{d i}^{\prime}$ & direct-axis transient reactance of Generator $i$ \\
$M_{i}$ & inertia of Generator $i$ \\
$D_{i}$ & damping coefficient of Generator $i$ \\
\hline
\end{tabular}

TABLE I

SYSTEM PARAMETERS

reduce the order of the interconnections and determine effective mutual couplings between the synchronous generators [8].

The relative normalized frequency of Generator $i$ is defined in this work as $\omega_{i}=\frac{\omega_{i}^{a c t}-\omega^{\text {nom }}}{\omega^{\text {nom }}}$, where $\omega^{\text {nom }}$ is the nominal angular frequency of the power system and $\omega_{i}^{\text {act }}$ is the actual angular frequency of Generator $i$. Let $\dot{\delta}_{i}$ and $\dot{\omega}_{i}$ denote the derivatives of $\delta_{i}$ and $\omega_{i}$ with respect to time, respectively. Then, assuming there is no power control in the power system, the swing equation for Generator $i$ in an interconnected system is expressed as [9], [10]

$$
\begin{aligned}
\dot{\delta}_{i} & =\omega_{i} \\
M_{i} \dot{\omega}_{i} & =-D_{i} \omega_{i}+\left(P_{m, i}-P_{e, i}\right) .
\end{aligned}
$$

where the electrical power of Generator $i$ is defined as [11]

$$
\begin{array}{cl}
P_{e, i}=\sum_{k=1}^{N} \quad & \left|E_{i}\right|\left|E_{k}\right|\left[G_{i k} \cos \left(\delta_{i}-\delta_{k}\right)+\right. \\
& \left.B_{i k} \sin \left(\delta_{i}-\delta_{k}\right)\right],
\end{array}
$$

where $G_{i k}=G_{k i} \geq 0$ is the Kron-reduced equivalent conductance between Generators $i$ and $k, B_{i k}=B_{k i}>0$ is the Kronreduced equivalent susceptance between Generators $i$ and $k$, and $Y_{i k}=G_{i k}+\sqrt{-1} B_{i k}$ is the Kron-reduced equivalent admittance between Generators $i$ and $k$. Let $P_{a, i}=P_{m, i}-P_{e, i}$ denote the accelerating power of Generator $i$, then the swing equation is represented as

$$
\begin{aligned}
& \dot{\delta}_{i}=\omega_{i} \\
& \dot{\omega}_{i}=\frac{1}{M_{i}}\left[-D_{i} \omega_{i}+P_{a, i}\right] .
\end{aligned}
$$

\section{Parametric Feedback Linearization Control}

Typically synchronous generators are equipped with power control schemes (such as exciter and governor controls) that help to adjust a generator's internal settings to respond to changes in the overall power grid. However, these local controllers are often insufficient due to their slow reaction to rapid systemwide changes. Thus, we develop a parametric feedback linearization (PFL) controller that does not assume the existence of any other local generator control; this assumption provides a more conservative picture of the power system stability. Consequently, if the proposed PFL controller can alone stabilize a generator after the occurrence of a disturbance in the system, it is more likely that the power system will be stabilized when both the PFL controller and the power controllers are activated in the system.

Without external power control, as described in Eq. (3), synchronous generators cannot alone achieve transient stability 
in the presence of a fault or when circuit breakers open beyond the critical clearing time either due to a malfunction or cyber attack. The use of external control, however, can help to achieve transient stability. Applying an external stabilizing power source at Generator $i$, termed as $U_{i}$, modifies the swing equation to

$$
\begin{aligned}
& \dot{\delta}_{i}=\omega_{i} \\
& \dot{\omega}_{i}=\frac{1}{M_{i}}\left[-D_{i} \omega_{i}+P_{a, i}+U_{i}\right] .
\end{aligned}
$$

Consequently, the proposed controller affects the dynamics of the power system by absorbing or injecting a specified amount of real power through the application of a fast-acting flywheel at the designated generator. A positive value of $U_{i}$ indicates that the controller of Generator $i$ is injecting power into the corresponding generator bus and a negative value implies that power is being absorbed from the generator bus.

\section{A. Frequency Stability}

The goal of the PFL controller is to asymptotically drive the frequency of the system generators into stability after the occurrence of a disturbance in the power grid; i.e., it is required that $\lim _{t \rightarrow \infty} \omega_{i}(t)=0 \forall i \in\{1, \ldots, N\}$ is achieved after the activation of the PFL controller.

Feedback linearization [12, Ch. 13] is one approach used to transform a nonlinear plant into an equivalent closed-loop linear system. One way to implement feedback linearization is by introducing a control signal that cancels out the nonlinear terms in the system dynamics such that the closed-loop system experiences (full or partial) linear dynamics. In order to fully cancel the nonlinear terms in the swing equation (i.e., the $\frac{P_{m, i}-P_{e, i}}{M_{i}}$ term) and enhance the system stability, we let the external stabilizing control be represented as

$$
U_{i}=-\left(P_{a, i}+F_{i} \omega_{i}\right),
$$

where $\left(D_{i}+F_{i}\right)>0$ and $F_{i} \geq 0$ is called the frequency stability parameter. Consequently, the swing equation of the interconnected power system (assuming exact knowledge of the system parameters), after implementing the PFL controller, reduces into a decoupled linear equation in the form of

$$
\dot{x}_{i}=A_{i} x_{i},
$$

where $x_{i}=\left[\begin{array}{c}\delta_{i} \\ \omega_{i}\end{array}\right]$ and $A_{i}=\left[\begin{array}{cc}0 & 1 \\ 0 & \frac{-1}{M_{i}}\left(D_{i}+F_{i}\right)\end{array}\right]$.

For this case, $x_{i}$ is called the state variable of Generator $i$. To verify the stability of the power system after implementing the proposed controller, the eigenvalues of $A_{i}$ are calculated and checked to determine if they lie in the left-hand complex plane. The eigenvalues of $A_{i}$ are found as 0 and $\frac{-1}{M_{i}}\left(D_{i}+F_{i}\right)$; thus, $\lim _{t \rightarrow \infty} \omega_{i}(t)=0$ is achieved [12, Theorem 4.5]. Consequently, the power system is stable under the proposed PFL controller. Because the frequency stability parameter appears to directly affect the value of the nonzero eigenvalue, it is expected that higher values of $F_{i}$ will drive the power system to stability faster; however, higher values of $F_{i}$ implies that the PFL controller would need higher amounts of external power.
Let $\boldsymbol{x}=\left[x_{1}, x_{2}, \ldots, x_{N}\right]^{T}$, where $(\cdot)^{T}$ is the transpose operator, denotes the system state variable, $\boldsymbol{A}=$ $\left[A_{1}, A_{2}, \ldots, A_{N}\right]^{T}$ represents the overall system matrix, and $\boldsymbol{U}=\left[U_{1}, U_{2}, \ldots, U_{N}\right]^{T}$ represents the controller output for the synchronous generators. Then, the dynamics of the overall power system (after implementing the PFL control) can be described as

$$
\dot{\boldsymbol{x}}=\boldsymbol{A} \boldsymbol{x} .
$$

\section{B. Phase Stability}

The development in Eq. (5) focuses on stabilizing the frequency of the system generators and promises no control over the phase of the system generators. However, to maintain a phase cohesiveness between the generators of the power system, the absolute difference between the phase of any two generators should be less than $100^{\circ}$ [2], [13].

In order to accomplish such phase cohesiveness, the PFL controller can be modified to account for the phase of the generators. Specifically, the PFL controller can be expressed as

$$
U_{i}=-\left(P_{a, i}+G_{i}\left(\delta_{i}-\delta_{i}^{*}\right)+F_{i} \omega_{i}\right),
$$

where $G_{i} \geq 0$ is called the phase stability parameter and $\boldsymbol{\delta}^{*}=$ $\left[\delta_{1}^{*}, \delta_{2}^{*}, \ldots, \delta_{N}^{*}\right]^{T}$ is the desired phase of the system generators. The $G_{i}\left(\delta_{i}-\delta_{i}^{*}\right)$ term will drive the PFL controller to settle the phase of the system generators on $\delta^{*}$. The values of $\delta^{*}$ are selected such that

$$
\left|\delta_{i}^{*}-\delta_{j}^{*}\right| \leq 100^{\circ}, \forall i, j \in\{1, \ldots, N\} .
$$

Consequently, phase cohesiveness is maintained during and after the controller's active time.

\section{Integration with Governor Control}

The PFL controller integrates naturally with the governor control commonly found in power systems. The governor control slowly adjusts the mechanical power of a generator in order to keep the frequency of the generator within the stability margin. However, although the PFL controller has a similar goal, the governor has a very slow time response.

One way to implement a governor controller is to slowly close the gap between the mechanical and the electrical powers of the generator. Mathematically, let $\dot{P}_{m, i}$ denote the derivative of $P_{m, i}$ with respect to time, then this implementation can be represented for Generator $i$ as

$$
\dot{P}_{m, i}=\kappa_{i}\left(P_{e, i}-P_{m, i}\right),
$$

where $\kappa_{i} \geq 0$. A value of $\kappa_{i}=0$ indicates that the governor control is not activated on Generator $i$.

\section{Features of the Proposed Controller}

Some of the advantages of the proposed stabilizing solution over previous work includes shorter transient stability time for the synchronous generators. The controller also integrates well with other power system controllers; for example, the governor control aligns naturally with the PFL controller. Thus, if the exact system parameters are unknown to the PFL controller, the governor can provide added robustness 

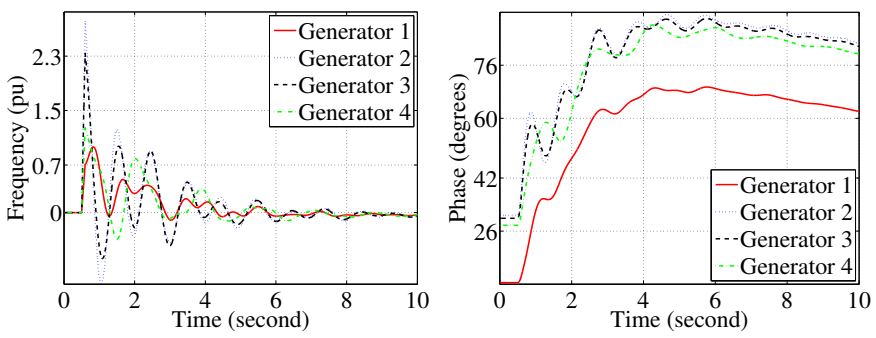

Fig. 2. System performance when only governor control is activated

against the parameter error. In addition, the design of the proposed controller is straightforward and is easy to implement. Further, the development of this stabilizing control provides a natural tool to demonstrate tradeoffs between the degree of available external power and the stability time of the synchronous generators. Moreover, the PFL controller does not need real-time updates about the system state information in order to effectively stabilize the power system; the controller can stabilize the power grid as long it obtains frequent and periodic updates from the system sensors though the smart grid communication network. The proposed controller can also easily be implemented in a step-wise manner.

\section{Numerical Results}

The New England 10-generator 39-bus power system of Fig. 1 is considered. The values of $M_{i}$ 's and $X_{d i}^{\prime}$ 's are found from [14], [15] and $D_{i}$ is set to $20 \mathrm{msec}$. The power system is assumed to be running in normal secure state from $t=0$ to $t=$ 0.5 seconds. However, a three-phase fault occurs at Bus 17 at $t=0.5$ seconds. Then, Line $17-18$ is tripped out to clear the fault at $t=0.6$ seconds. Finally, the PFL controller is activated on all generators at $t=0.7$ seconds.

Before the occurrence of the 3-phase fault, load flow analysis of the power system is conducted to find the values of $P_{e, i}, \delta_{i}$, and $\left|E_{i}\right|$ for each generator. Because the power system is balanced and there are no transients (before the fault), the mechanical power of each generator also equals the electrical power of that generator at the moment of fault.

For the following numerical results, the stability time of a generator is calculated by finding the controller's active time after which the frequency of the generator is restricted to a $2 \%$ threshold. In other words, stability time of Generator $i$ is the time it takes the controller to keep the frequency stable (i.e., $\left|\omega_{i}\right| \leq 0.02$ ) permanently. Further, the following figures show the performance results for the first four synchronous generators; however, similar behaviour can be found for the rest of the generators.

\section{A. Power System without PFL Control}

The performance of the power system when only a governor control is activated is shown in Fig. 2. The implemented governor slowly adjusts the value of the mechanical power of a generator in order to reduce the gap between $P_{m}$ and $P_{e}$ so that the change in frequency is slowed (and ultimately reversed back into stability). The implemented nonlinear governor closes $90 \%$ and $99 \%$ of the gap between $P_{m}$ and $P_{e}$ in 3.07 and 6.14 seconds, respectively. Results of Fig. 2 show that the
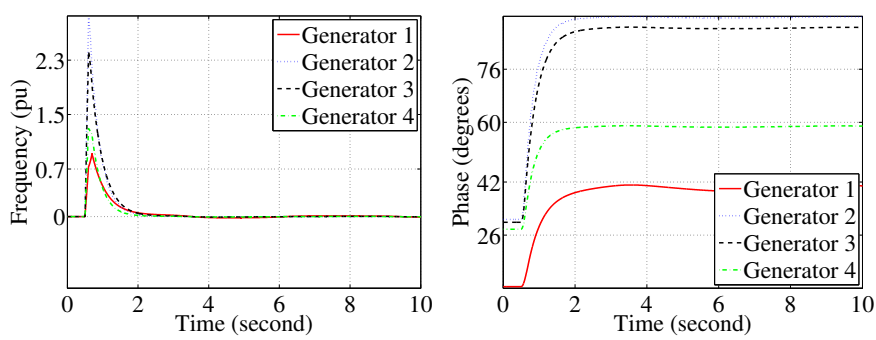

Fig. 3. System performance when only PFL control is activated
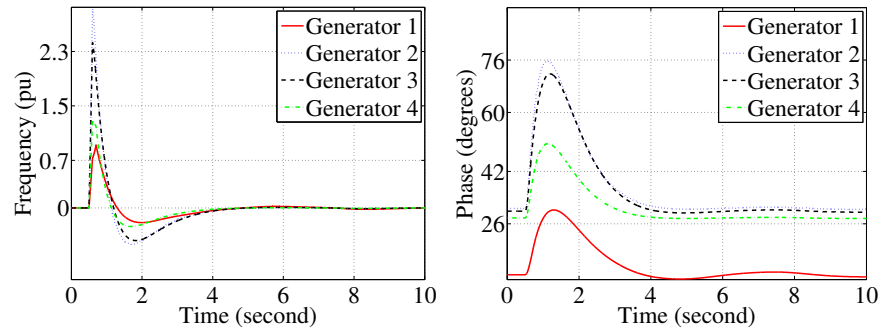

Fig. 4. System performance when phase stability parameter is activated

governor slows down the instability of the system; however, it needs a relatively long time (around 17.5 seconds) to bring the generators' relative normalized frequency to within the stability margin of $\pm 0.02 \mathrm{pu}$.

\section{B. Frequency Stability}

Fig. 3 displays the effect of implementing the proposed PFL controller on the performance of the power system. Both the governor control and the phase stability are not incorporated in Fig. 3 to emphasize the results of using the proposed controller in stabilizing the frequency of the system generators. The PFL controller is designed to access the system state variable (i.e., $\boldsymbol{x})$ every $10 \mathrm{msec}$, and the value of the frequency stability parameter $\left(F_{i}\right)$ in Eq. (5) is set to $500 \mathrm{msec}$ for all generators. Further, the absolute value of any $U_{i}$ cannot exceed 5 pu.

It is noted that the power system achieves frequency stability within a short time. For example, stability time of Generator 1 is about 2.34 seconds. It is to be noted that because the mechanical power of the synchronous generators does not change in this case, the stabilizing controller compensates the difference between $P_{m}$ and $P_{e}$ in order to keep the power system stable.

\section{Frequency and Phase Stability}

The phase stability parameter is activated in this case. As a numerical example, let the values of $\boldsymbol{\delta}^{*}$ in Eq. (8) be equal to the phase of the system generators before the occurrence of the three-phase fault at Bus 17; i.e., the controller will drive the phase of the generators to the before-fault values. Let the values of $F_{i}$ and $G_{i}$ be both set to 0.5 .

Fig. 4 shows the effect of activating the phase stability parameter in the PFL controller. It is observed that the phase of the system generators is controlled as promised by Eq. (8). In this case, the stability time of Generator 1 is around 5.53 seconds, which is slightly higher than that of the case when phase stability parameter is not activated. However, the extra time is needed by the PFL controller in order to 


\begin{tabular}{|l||l|l|l||l|l|l|}
\hline Gen. & $\begin{array}{l}\text { Governor } \\
\text { Control }\end{array}$ & $\begin{array}{l}\text { Flocking } \\
\text { Control }\end{array}$ & $\begin{array}{l}\text { CPI } \\
\text { Control }\end{array}$ & $\begin{array}{l}\text { PFL } \\
\text { Control }\end{array}$ & $\begin{array}{l}\text { PFL \& } \\
\text { Phase }\end{array}$ & $\begin{array}{l}\text { PFL \& } \\
\text { Governor }\end{array}$ \\
\hline 1 & 17.5268 & 47.1266 & 4.9270 & 2.3425 & 5.5332 & 1.6180 \\
2 & 17.3304 & 45.3626 & 5.0617 & 1.4841 & 3.6801 & 1.5417 \\
3 & 17.3456 & 45.3269 & 5.0856 & 1.7205 & 3.6628 & 1.7633 \\
4 & 17.6166 & 47.0708 & 5.2622 & 1.2634 & 3.2608 & 1.1706 \\
5 & 17.6046 & 45.3384 & 5.3558 & 1.0793 & 2.9979 & 0.9920 \\
6 & 17.6146 & 46.9529 & 5.2447 & 1.4792 & 3.3510 & 1.4040 \\
7 & 17.6139 & 45.3225 & 5.2533 & 1.1866 & 3.1868 & 1.0831 \\
8 & 17.5799 & 47.0606 & 5.0235 & 1.2632 & 3.2148 & 0.9823 \\
9 & 17.6114 & 45.3489 & 5.2276 & 1.4441 & 3.2510 & 1.3188 \\
\hline
\end{tabular}

Fig. 5. Stability time (second)

achieve both the desired phase cohesiveness and the frequency stability.

\section{Comparison with Recent Work}

As a comparison, the performance of the recently proposed flocking control and consensus proportional integral control schemes is investigated next.

Flocking control was proposed in [1]-[3] to address stability issues in power system after the clearance of a fault. In general, a flocking scheme can be used to control flock-mates (or agents) to achieve flock centering where an agent stays close to nearby agents, collision avoidance where an agent avoids colliding with nearby agents, and velocity matching where an agent attempts to match the speed of nearby agents [16]-[18]. For a power system application, the flock centring feature can be used to achieve phase angle cohesiveness while the velocity matching feature can be used to achieve frequency synchronization between the generators of the power system.

Andreasson et al. proposed to use the CPI controller in order to achieve automatic frequency control (through affecting the mechanical power of the generators); however, the PFL controller affects the output of fast-acting external power sources instead of changing the mechanical power of the designated generator. Consequently, to be consistent with the PFL and flocking controls, the numerical results of the CPI scheme used the output of the CPI controller to affect the output of the fast-acting flywheels. As a summary of the different control schemes, a detailed record of the stability time of each generator (except Generator 10) in the New England power system is shown in Fig. 5. Compared to the governor or flocking controls, the PFL controller achieves the quickest stability. However, activating the phase stability parameter increases the stability time slightly. Further, savings in system stability can be accomplished by activating both PFL and governor controls at the same time. Moreover, the PFL controller outperforms the recently-proposed centralized CPI controller for comparable parameters.

\section{CONCLusions}

This paper proposes a parametric frequency and phase stabilizing controller for smart grid systems under severe fault or malfunction of protection devices. System state information is collected by sensors and transmitted through a communication network to distributed controllers. Based on the received data, a feedback linearization control is applied using fast-acting flywheels situated near the synchronous generators to balance the swing equation and drive the power system to stability.

System performance is investigated when the proposed controller is applied to the New England 39-bus 10-generator power system. Further, the performance is studied when both the proposed and governor controls are activated in the power system. Results of this work show the effectiveness of the proposed controller in stabilizing the power grid and making it more resilient to disturbance.

\section{ACKNOWLEDGMENTS}

This work was supported by the National Science Foundation under grant ECCS-1028246 and the Natural Sciences and Engineering Research Council of Canada.

\section{REFERENCES}

[1] J. Wei, D. Kundur, and T. Zourntos, "On the Use of Cyber-Physical Hierarchy for Smart Grid Security and Efficient Control," in IEEE Canadian Conference on Electrical and Computer Engineering (CCECE), pp. 1-6, April/May 2012.

[2] J. Wei, D. Kundur, T. Zourntos, and K. L. Butler-Purry, "A FlockingBased Dynamical Systems Paradigm for Smart Power System Analysis," in IEEE Power and Energy Society General Meeting (PESGM), pp. 1-8, July 2012.

[3] J. Wei and D. Kundur, "Two-Tier Hierarchical Cyber-Physical Security Analysis Framework For Smart Grid," in IEEE Power and Energy Society General Meeting (PESGM), pp. 1-5, July 2012.

[4] M. Andreasson, H. Sandberg, D. V. Dimarogonas, and K. H. Johansson, "Distributed Integral Action: Stability Analysis and Frequency Control of Power Systems," in IEEE Annual Conference on Decision and Control (CDC), pp. 2077-2083, December 2012.

[5] M. Andreasson, D. V. Dimarogonas, K. H. Johansson, and H. Sandberg, "Distributed vs. Centralized Power Systems Frequency Control," in European Control Conference (ECC), pp. 3524-3529, July 2013.

[6] M. Andreasson, D. V. Dimarogonas, H. Sandberg, and K. H. Johansson, "Distributed Control of Networked Dynamical Systems: Static Feedback and Integral Action and Consensus," IEEE Transactions on Automatic Control, vol. 59, pp. 1750-1764, July 2014.

[7] J. W. Chapman, M. D. Ilic, C. A. King, L. Eng, and H. Kaufman, "Stabilizing a Multimachine Power System via Decentralized Feedback Linearizing Excitation Control," IEEE Transactions on Power Systems, vol. 8, pp. 830-839, August 1993.

[8] F. Dörfler and F. Bullo, "Kron Reduction of Graphs With Applications to Electrical Networks," IEEE Transactions on Circuits and Systems I: Regular Papers, vol. 60, pp. 150-163, January 2013.

[9] P. M. Anderson and A. A. Fouad, Power System Control and Stability. IEEE Power Systems Engineering Series, IEEE Press, 1994.

[10] F. Dörfler and F. Bullo, "Synchronization and Transient Stability in Power Networks and Non-Uniform Kuramoto Oscillators," in American Control Conference (ACC), pp. 930-937, June/July 2010.

[11] A. R. Bergen and V. Vittal, Power Systems Analysis. Prentice-Hall, second ed., 2000.

[12] H. K. Khalil, Nonlinear Systems. Prentice-Hall, third ed., 2002.

[13] P. W. Sauer and M. A. Pai, Power System Dynamics and Stability. Prentice-Hall, 1998.

[14] T. Athay, R. Podmore, and S. Virmani, "A Practical Method for the Direct Analysis of Transient Stability," IEEE Transactions on Power Apparatus and Systems, vol. 98, pp. 573-584, March/April 1979.

[15] B. Pal and B. Chaudhuri, Robust Control in Power Systems. Power Electronics and Power Systems Series, Springer, 2006.

[16] C. W. Reynolds, "Flocks, Herds, and Schools: A Distributed Behavioral Model," Computer Graphics - Annual Conference on Computer Graphics and Interactive Techniques, vol. 21, pp. 25-34, July 1987.

[17] R. Olfati-Saber, "Flocking for Multi-Agent Dynamic Systems: Algorithms and Theory," IEEE Transactions on Automatic Control, vol. 51, pp. 401-420, March 2006.

[18] R. Olfati-Saber, J. A. Fax, and R. M. Murray, "Consensus and Cooperation in NetworkedMulti-Agent Systems," Proceedings of the IEEE, vol. 95, pp. 215-233, January 2007. 